\title{
Predicting Neck Fluid Accumulation While Supine
}

\author{
Daniel Vena, MHSc ${ }^{1,2}$; Babak Taati, PhD $^{1,3}$ and Azadeh Yadollahi, PhD ${ }^{1,2^{*}}$ \\ ${ }^{1}$ University Health Network - Toronto Rehabilitation Institute, Toronto, Canada \\ ${ }^{2}$ Institute of Biomaterials and Biomedical Engineering, \\ University of Toronto, Toronto, Canada \\ ${ }^{3}$ Department of Computer Science, University of Toronto, Toronto, Canada
}

Submitted May 2015. Accepted for publication July 2015.

\begin{abstract}
When lying supine, fluid shifts rostrally from the legs and accumulates in the neck, which is a risk factor for obstructive sleep apnea. The objective of this study was to model neck fluid accumulation using one-time baseline measurements of body fluid, demographics, and anthropometrics. Using bioelectrical impedance, leg and neck fluid volumes (LFV and NFV) were measured continuously and simultaneously. Thirty non-obese adults (13 men) stood quietly for 5 minutes, and then lay supine for 90 minutes while fluid volumes were measured. Neck circumference $(\mathrm{NC})$ was measured before and after the supine period. Results demonstrated that, compared to women, men experienced a greater increase in NC after lying supine. Furthermore, baseline LFV at the onset of lying supine was significantly correlated with $\triangle \mathrm{LFV}(\mathrm{r}=0.44$, $\mathrm{p}=0.014)$ and $\Delta \mathrm{NC}(\mathrm{r}=0.51, \mathrm{p}=0.008)$ after 90 minutes supine. The findings identify that sex and baseline LFV predict both the fluid leaving the legs and increase in NC during recumbency.
\end{abstract}

Keywords: Rostral fluid shift, leg fluid volume, neck fluid volume, supine position, upper airway

\section{INTRODUCTION}

Overnight neck fluid accumulation is a risk factor for upper airway pathologies and respiratory disorders [1]-[4]. The accumulation of neck fluid could cause distension of the neck veins and/or edema formation in the pharyngeal soft tissue. These changes increase pressure on the upper airway and could cause the narrowing of the upper airway $[3,5,6]$, increase upper airway resistance [1,7] and collapsibility $[4,8]$, and increase the severity of obstructive sleep apnea (OSA) [9]. Understanding the factors that increase the likelihood of fluid accumulation in the neck can help identify patients at risk for fluid related respiratory disorders and also identify avenues for new therapies that could target these factors. The redistribution of fluid from the legs into the upper body while recumbent is an important mechanism through which fluid may accumulate in the neck. It has been demonstrated that the volume of fluid leaving the legs while recumbent is correlated with increased neck circumference (NC) signaling the accumulation of fluid in the neck $[2,10,11]$.

*Corresponding author: Azadeh Yadollahi, University Hospital Network - Toronto Rehabilitation Institute, Room 12-106, 550 University Ave., Toronto, ON, M5G 2A2, Canada. Telephone: 416-597-3422 x7936. Fax: 416-597-8959.Email: azadeh.yadollahi@uhn.ca. Other authors: daniel.vena@uhn.ca, babak.taati@uhn.ca. 
The effects of gravity and Starling's forces cause fluid redistribution during the transition from a standing to a recumbent position. Based on the Starling model, the balance of hydrostatic and oncotic forces controls the fluid movement between the capillaries and the interstitial spaces [12]. When hydrostatic pressure is higher in the capillary spaces compared to the interstitial spaces, e.g., in sitting or standing postures, the resulting pressure gradient promotes the movement of blood plasma out of the capillaries and into the interstitial spaces [13]. Therefore, these postures result in an increased overall leg fluid volume (LFV) between 100 and $300 \mathrm{ml}$ [14-16]. When lying down, the reverse occurs; hydrostatic pressure in the capillaries of the legs is reduced, allowing interstitial fluid to be reabsorbed back into the venous system [17-20], which moves rostrally through the vascular system to the upper body due to gravity. The result is reduced fluid volume in the legs and associated increases in fluid volumes of the thorax and head [18, 19].

Identifying patients that are susceptible to increased neck fluid accumulation after prolonged recumbency can be a useful predictor of individuals at risk of upper airway pathologies due to fluid shift. However, measuring the rostral shift of fluid from the legs to the neck during prolonged recumbency is inconvenient and time consuming. It either requires data collection for hours or overnight [2], or the use of anti-shock trousers to apply lower body positive pressure to quicken LFV shift and simulate prolonged rostral fluid shift [1], which can be cumbersome. Therefore, it is important to investigate simple measurements that can be performed quickly and conveniently (e.g., in clinic), which in turn could predict the amount of fluid that would accumulate in the neck after prolonged recumbency. Throughout the paper, we refer to these instantaneous, one-time measurements taken before prolonged recumbency as baseline measures.

Previous studies have shown that in heart failure patients, an increased baseline leg edema score is significantly correlated with the overnight change in LFV and the increase in NC [11,21]. However, leg edema score is determined subjectively; it involves the application of pressure to a small area on the lower leg to create an indentation in the tissue. A subjective score from 1+ (less severe) to 4+ (more severe) is given based on the depth of indentation and how long the indentation remains [22]. In addition, these studies only focused on heart failure patients experiencing fluid overload.

The current study explored various baseline metrics measured objectively to improve prediction of neck fluid accumulation in the general population. These metrics included: baseline LFV, NC, neck fluid volume (NFV), and upper-airway crosssectional area (UAXSA). Furthermore, in past studies, neck fluid accumulation had only been measured using the change in $\mathrm{NC}$ as a surrogate measure [2, 10, 11]. In this study, the bioelectrical impedance method was used to simultaneously measure both LFV and NFV [23]. The purpose of the present study was to develop a model based on baseline measurements of body fluid, as well as demographics and anthropometrics of the participants to predict fluid accumulation in the neck.

\section{MATERIALS AND METHODS}

\subsection{Participants}

The protocol was approved by the Research Ethics Board of Toronto Rehabilitation Institute, and all participants provided written informed consent prior to participating in the study. Criteria for inclusion were men and women between 18 and 65 years of age 
with a body mass index (BMI) $<30 \mathrm{~kg} / \mathrm{m}^{2}$, and a blood pressure $\leq 140 / 90 \mathrm{mmHg}$. Women were included only if they were premenopausal and did not have their menstrual period at the time of experiments. The exclusion criteria were a history of hysterectomy, having metal implants, history of cardiovascular, renal, or respiratory diseases, use of prescribed medications for those diseases, or taking any over the counter medication that might influence fluid retention, such as diuretics or nonsteroidal anti-inflammatory agents. Participants were instructed to avoid the intake of alcohol or caffeine on the day of the study. Participants were recruited from the community by advertisement.

\subsection{Neck Circumference, Upper-Airway Cross-Sectional Area, and Airway Length Measurements}

At the beginning of each session, a tape measure was used to measure $\mathrm{NC}$ just above the cricothyroid cartilage. A line was drawn at this level to ensure the NC measurement at the end of the experiment was made at the same level, as described in past studies [4,21]. Upper-airway length ( $\mathrm{L}_{\mathrm{UA}}$, distance from velum to glottis) and the average UAXSA from velum to glottis were measured using acoustic pharyngometry [24].

\subsection{Leg and Neck Fluid Volumes Measurements}

Fluid was measured in the leg and neck using bioelectrical impedance, a non-invasive technique used to estimate the fluid volume of tissues. The bioelectrical impedance method of fluid measurement is well validated and highly reproducible with an accuracy of $0.5 \%$ compared to reference measures of total body water, repeatability within $0.3 \%$, and test-retest correlation $>95 \%[25,26]$.

The method is based on Ohm's law (V = IR), where the resistance of a tissue to electrical current is inversely related to its fluid content and directly related to its length: $\mathrm{R}=\mathrm{rL}^{2} / \mathrm{v}$, where $\mathrm{r}$ is the resistivity of the fluid, $\mathrm{L}$ is the segment's length, $\mathrm{v}$ is the fluid volume, and $\mathrm{R}$ is resistance [27-29]. The resulting equation $\left(\mathrm{v}=\mathrm{rL}^{2} / \mathrm{R}\right)$ has been widely used to estimate total body water, where L is replaced with the subject's height $[25,26]$. Past studies have also used bioelectrical impedance to measure the fluid volume of individual body segments. In these studies, segments are assumed to be cylindrical in shape, with L representing the length of the segment [30-33]. In a previous study, we developed a system for continuous measurement of fluid volumes in various body segments [34]. We used a modified version of this equation to reflect the tapered shape of the body segments (leg, abdomen, chest, and neck). Therefore, fluid volume was estimated as [35], [36]:

$$
v=\frac{\rho^{2 / 3}}{3(4 \pi)^{1 / 3}}\left(\frac{L}{C_{1} C_{2} R}\right)^{2 / 3} L\left(C_{1}^{2}+C_{2}^{2}+C_{1} C_{2}\right)
$$

where $C_{1}$ and $C_{2}$ are the top and bottom circumferences of the segment, respectively, $L$ is the segment's length, $\mathrm{R}$ is the segment's resistance, and $\rho$ is blood resistivity, which is estimated as $47 \Omega \mathrm{cm}[29]$. 


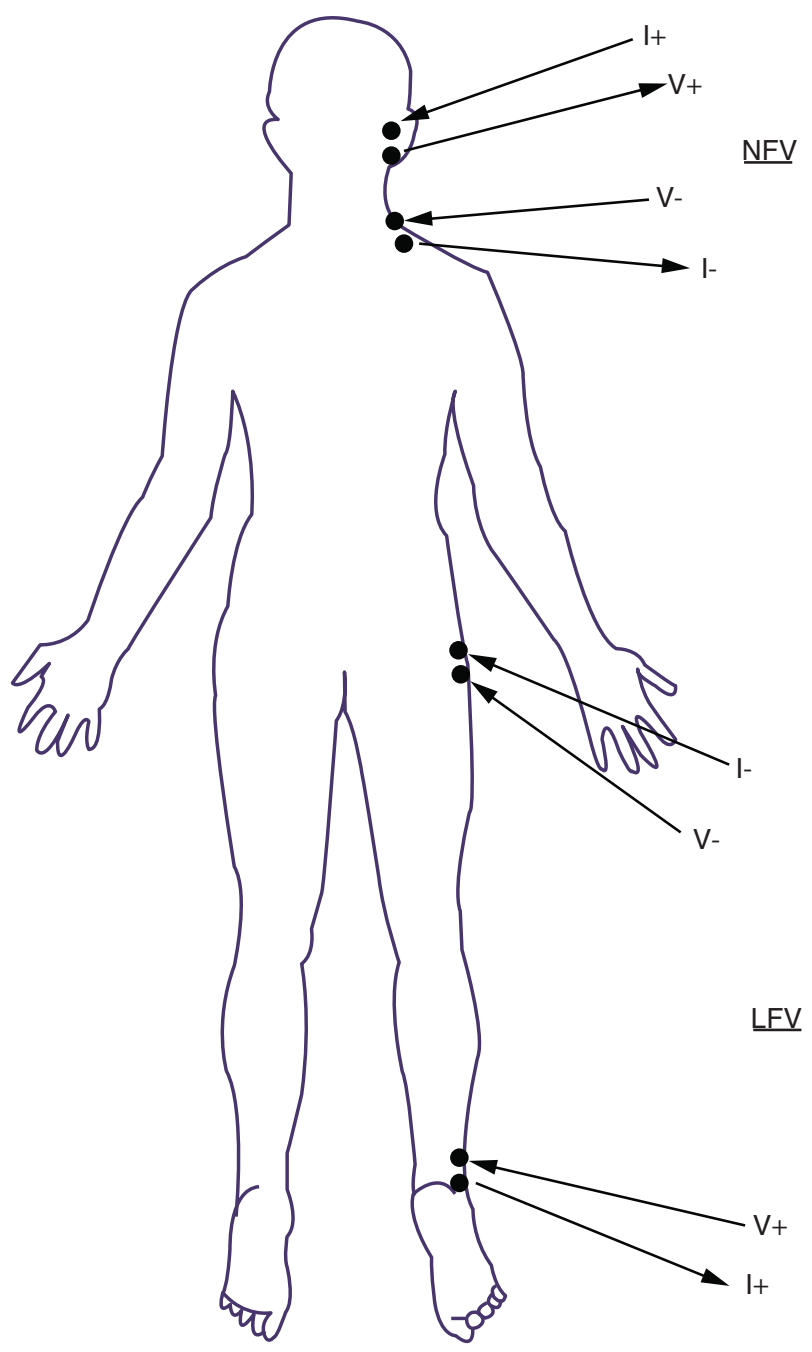

Figure 1. Positions of the surface electrodes for recording the bioelectrical impedance in the leg and neck. I+ and I- denote surface electrodes used to inject a low amplitude (400 $\mu \mathrm{a})$, high frequency $(25 \mathrm{kHz}$ and $50 \mathrm{kHz}$ for the leg and neck, respectively) current. V+ and V- denote surface electrodes used to measure the voltages across the leg and neck segments. LFV denotes leg fluid volume and NFV denotes neck fluid volume.

For both the leg and the neck, two surface electrodes were used to inject a low amplitude $(400 \mu \mathrm{a})$, high frequency current $(25 \mathrm{kHz}$ and $50 \mathrm{kHz}$ for the leg and neck, respectively) and two surface electrodes were used to measure the voltage (Figure 1) to estimate bioelectrical impedance of the segment ( $R$ in eqn. 1) [34]. In Figure 1, electrodes to inject current are denoted as I- and I+ and electrodes to measure voltage 
are denoted as $\mathrm{V}$ - and $\mathrm{V}+$. Voltage measuring electrodes were placed at the ankle and upper thigh of the right leg for the LFV measurement. To measure NFV, voltagemeasuring electrodes were placed on the right side of the neck below the right ear and at the base of the neck (Figure 1). The current-injecting electrodes were placed one inch away from the voltage-measuring electrodes. To isolate the signal from different body segments for bioelectrical impedance measurements, a different injecting frequency was used for the leg and neck. The electrodes were secured to the skin using adhesive tape. At the beginning of the study, length and circumference of each segment were measured with a measuring tape at the level of the voltage-measuring electrodes. For NFV, the NC measurement was used for both circumferences $\mathrm{C}_{1}$ and $\mathrm{C}_{2}$ (eqn. 1).

\subsection{Protocol}

This study is part of a previous study [34] conducted at the Toronto Rehabilitation Institute. While seated, blood pressure was measured to ensure subjects were normotensive. Next, surface electrodes were applied to the participant, as shown in Figure 1, to measure the bioelectrical impedance. All experiments were performed in the same room with the temperature maintained between $22^{\circ} \mathrm{C}$ and $24^{\circ} \mathrm{C}$. Next, participants were asked to stand motionless for 5 minutes. For the following 90 minutes, the subjects then lay awake in the supine position on a bed without a pillow. Bioelectrical impedance of the leg and neck were recorded continuously and simultaneously during both standing and supine positions. Subjects were instructed to remain motionless during the recordings.

\subsection{Baseline and Outcome Variables}

Baseline variables were selected as quantities that could be easily measured and represent fluid content in the body and/or have been shown to have a strong correlation with increases in NC while supine. The variables included were LFV after standing for 5 minutes $\left(\mathrm{LFV}_{\mathrm{st}}\right)$, NFV after standing for 5 minutes $\left(\mathrm{NFV}_{\mathrm{st} 5}\right), \mathrm{LFV}$ at baseline supine $\left(\mathrm{LFV}_{\mathrm{sp} 1}\right), \mathrm{NFV}$ at baseline supine $\left(\mathrm{NFV}_{\mathrm{sp} 1}\right)$, immediate change in LFV upon transitioning from standing to supine position $\left(\Delta \mathrm{LFV}_{\mathrm{P}}=\mathrm{LFV}_{\mathrm{sp} 1}-\mathrm{LFV}_{\mathrm{st} 5}\right)$, immediate change in NFV upon transitioning from standing to supine position $\left(\Delta \mathrm{NFV}_{\mathrm{P}}=\mathrm{NFV}_{\mathrm{sp1} 1}-\mathrm{NFV}_{\mathrm{st}}\right), \mathrm{NC}$ measured at baseline supine $\left(\mathrm{NC}_{\mathrm{sp1}}\right)$, upper-airway cross-sectional area at baseline supine (UAXSA $_{\mathrm{sp} 1}$ ), and length of the upper-airway (between the velum and the glottis) measured at baseline supine $\left(\mathrm{L}_{\mathrm{UA}}\right)$. Also computed was $\Delta \mathrm{LFV}_{90}\left(\Delta \mathrm{LFV}_{90}=\mathrm{LFV}_{\mathrm{sp} 90}-\right.$ $\mathrm{LFV}_{\text {sp1 } 1}$ ), where $\mathrm{LFV}_{\text {sp90 }}$ is the LFV after 90 minutes supine. Since previous studies have shown strong correlations between the change in LFV and change in NC [1]-[4], $\Delta \mathrm{LFV}_{90}$ was included to correlate independently with the outcome variables and to compare our results with those of the previous studies; however, it was not included in the multiple regression models, as discussed later. Lastly, demographic information including age, sex, height, weight, and BMI were also included in the regression model. Sex was defined as a binary variable with 0 and 1 representing men and women, respectively.

Outcome variables were selected as $\Delta \mathrm{NC}_{90}=\mathrm{NC}_{\mathrm{sp} 90}-\mathrm{NC}_{\mathrm{sp} 1}, \Delta \mathrm{NFV}_{90}=\mathrm{NFV}_{\mathrm{sp} 90}-$ $\mathrm{NFV}_{\mathrm{sp} 1}$, and $\triangle \mathrm{UAXSA}_{90}=\mathrm{UAXSA}_{\mathrm{sp} 90}-\mathrm{UAXSA}_{\mathrm{sp} 1}$, where $\mathrm{NC}_{\mathrm{sp} 90}, \mathrm{NFV}_{\mathrm{sp} 90}$, and UAXSA $_{\mathrm{sp} 90}$ were the NC, NFV and UAXSA after lying supine for 90 minutes, respectively. 


\subsection{Data Analysis}

The analysis was performed in three steps. In the first step, individual correlations were analyzed between each of the baseline variables and the outcome variables using Pearson correlations with normally distributed data, and Spearman's rank correlation with non-normally distributed data. Normality of the data was determined using the Kolmogorov-Smirnov test. A correlation was considered significant with a two-sided p-value $<0.05$.

In the second step of the analysis, a multiple linear regression model was developed to determine the most significant factors that contributed to the changes in outcome variables. Baseline variables were selected for the model using a forward stepwise selection. Using this approach, the model starts with no variables from the variable set, adding a variable if their associated p-value is below 0.05 , and removing variables if their $\mathrm{p}$-value is above 0.10 . The variable set included the entire baseline variable set already described, excluding $\Delta \mathrm{LFV}_{\mathrm{P}}$ and $\Delta \mathrm{NFV}_{\mathrm{P}}$, since they are linear combinations of the baseline leg and neck fluid volume measurements in the supine and standing position. We also excluded weight and height in the same variable set as BMI, given that BMI is a function of weight and height.

The final step of the analysis was to investigate whether the subjects can be classified into two groups of "high risk" and "low risk" regarding the effects of fluid accumulation in the neck. To do this, input variables were converted into standardized z-scores and a method based on the combination of principal component analysis (PCA) and unsupervised clustering was implemented. PCA is a statistical method that converts a set of correlated variables into a new set of uncorrelated (orthogonal) variables called principal components [37]. The principal components are linear combinations of the original variables weighted by their contribution to explaining the variance in a particular orthogonal dimension. PCA was used to reduce the dimensionality of an input variable set and to identify a subset of input variables that account for most of the variability in the data. The number of components to retain was selected based on the point on inflexion on the Scree plot (Figure 2a) which plots the component number versus the Eigenvalue as the dependent variable. The Eigenvalue is a scalar indicator of the substantive importance of the associated component with larger Eigenvalues representing more important components. Components were retained if they were to the left of the point of inflexion, but not including the inflexion point itself. We applied a similar approach for selecting input variables by plotting the factor weightings of each input variable in descending order (Figure 2b). A point of inflexion was identified and variables to the left of the point of inflexion were retained as a variable subset for further clustering analysis.

An unsupervised K-means clustering was then applied to the selected subset of variables to cluster the subjects into two groups $(K=2)$. This clustering method separates the data into $\mathrm{K}$ groups by establishing a centroid for each group. The position for the centroid is initialized and data points are assigned to the closest centroid (the assignment step). The centroid is then repositioned to minimize the within-cluster sum of squares (the update step). The assignment step and the update step are repeated until convergence; when the assignments no longer change [38]. Outcome variables were 
(a)

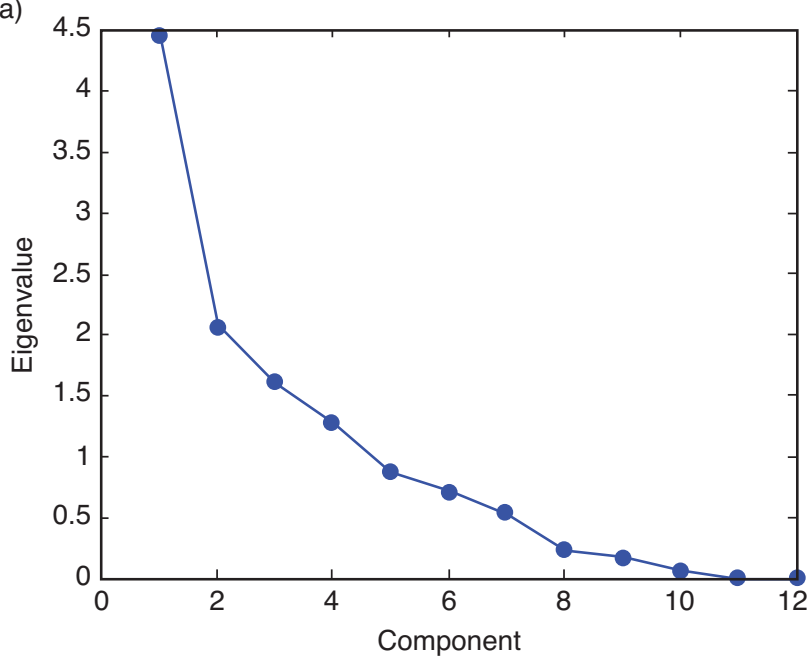

(b)

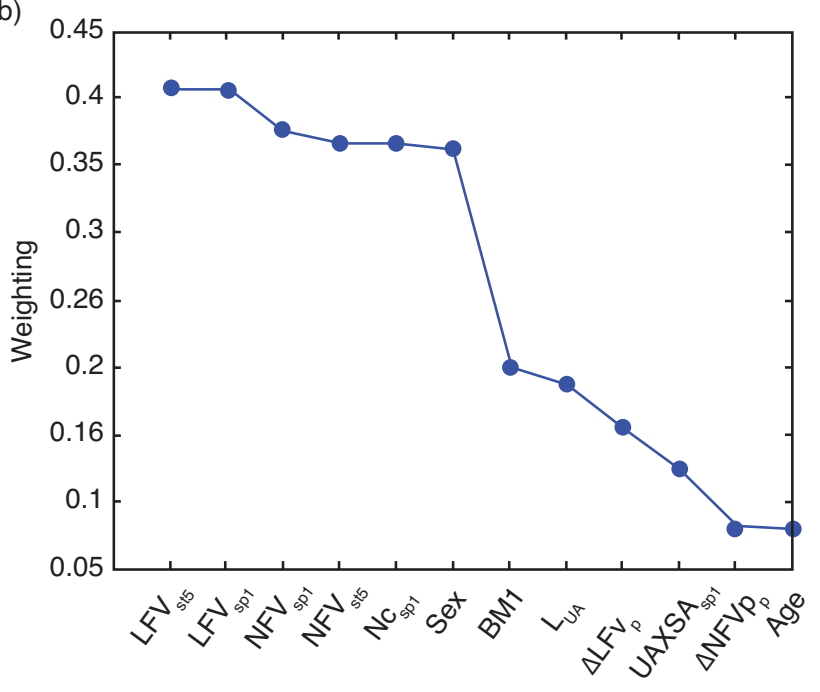

Variable

Figure 2. (a) Scree plot of the Eigenvalues of each component used to identify the inflexion point used as a threshold for the number of components to retain. The point of inflexion is located at Component 2, which means that only the first (Component) is retained. (b) A modified scree plot of the variables and the weightings of the variables on the first component, similarly used to identify the point of inflexion as a threshold for number of variables to retain. The inflexion point is located at the BMI variable, meaning all variables to the left of BMI are retained. 
compared between the two clusters using the student t-test for normally distributed data and the Kolmogorov-Smirnov test for non-normally distributed data; a two-sided pvalue of less than 0.05 was considered significant. Statistical analysis was performed using SAS 9.3 (SAS Institute Inc., Cary, NC), and PCA and clustering were performed in Matlab (MathWorks, Natick, MA).

\section{RESULTS}

Fifty-two candidates consented to participate in the study. One participant declined to continue after being instrumented. Eleven participants had movement artifacts in the bioelectrical impedance signals and their data were excluded. Ten participants did not have standing data collected and were excluded from this analysis. After all exclusions, a total of 30 participants (13 men and 17 women) were included in the analysis. Baseline fluid measures, as well as anthropometric data and variables for men, women, and all subjects are shown in Table 1. After lying supine for 90 minutes, NFV and NC in all subjects increased significantly $(\mathrm{p}<0.0001)$, while the upper-airway crosssectional area reduced significantly $(\mathrm{p}=0.036)$. Compared to men, women had smaller weight, height, baseline NC and UA length, but had similar age and BMI. In addition, women had lower baseline NFV and LFV at standing and supine. However, both experienced a similar change in LFV over the 90-minute supine period. In terms of

Table 1. Baseline and outcome variable data in all subjects, and associated p-values for statistical differences between men and women

\begin{tabular}{|c|c|c|c|c|}
\hline Variable & All & $\operatorname{Men}(n=13)$ & Women $(n=17)$ & $\begin{array}{c}\text { P-value } \\
\text { between } \\
\text { sexes }\end{array}$ \\
\hline Age, years & $39.2 \pm 11.7$ & $37.8 \pm 13.2$ & $39.2 \pm 11.7$ & 0.771 \\
\hline BMI, $\mathrm{kg} / \mathrm{m}^{2}$ & $23.3 \pm 2.9$ & $25.0 \pm 4.3$ & $22.8 \pm 2.6$ & 0.771 \\
\hline Weight, kg & $69.0 \pm 11.8$ & $81.7 \pm 15.7$ & $63.0 \pm 9.5$ & $<0.001$ \\
\hline Height, $\mathrm{cm}$ & $172.0 \pm 10.7$ & $180.7 \pm 9.3$ & $165.9 \pm 7.5$ & $<0.001$ \\
\hline $\mathrm{NC}_{\mathrm{sp} 1}, \mathrm{~cm}$ & $36.6 \pm 3.8$ & $41.0 \pm 3.4$ & $34.0 \pm 2.0$ & $<0.001$ \\
\hline $\mathrm{UAXSA}_{\mathrm{sp} 1}, \mathrm{~cm}^{2}$ & $2.9 \pm 1.4$ & $3.0 \pm 1.5$ & $2.7 \pm 1.1$ & 0.242 \\
\hline $\mathrm{NFV}_{\text {st5 }}, \mathrm{ml}$ & $223.9 \pm 60.2$ & $253.8 \pm 72.5$ & $200.7 \pm 26.6$ & 0.009 \\
\hline $\mathrm{LFV}_{\mathrm{st} 5}, \mathrm{ml}$ & $2195.5 \pm 288.9$ & $2532.2 \pm 482.6$ & $2057.5 \pm 256.4$ & 0.006 \\
\hline$\Delta \mathrm{NFV}_{\mathrm{P}}, \mathrm{ml}$ & $12.1 \pm 8.9$ & $11.0 \pm 12.5$ & $11.9 \pm 7.6$ & 0.685 \\
\hline$\Delta \mathrm{LFV}_{\mathrm{P}}, \mathrm{ml}$ & $66.7 \pm 28.7$ & $59.6 \pm 51.5$ & $68.0 \pm 21.0$ & 0.415 \\
\hline $\mathrm{NFV}_{\mathrm{sp} 1}, \mathrm{ml}$ & $236.0 \pm 60.5$ & $264.8 \pm 71.9$ & $212.6 \pm 28.1$ & 0.006 \\
\hline $\mathrm{LFV}_{\mathrm{sp} 1}, \mathrm{ml}$ & $2128.8 \pm 278.7$ & $2472.7 \pm 499.2$ & $1989.8 \pm 246.4$ & 0.002 \\
\hline$\Delta \mathrm{LFV}_{90}, \mathrm{ml}$ & $143.7 \pm 37.3$ & $160.9 \pm 56.6$ & $138.9 \pm 32.4$ & 0.182 \\
\hline $\mathrm{L}_{\mathrm{UA}}, \mathrm{cm}$ & $8.8 \pm 1.5$ & $10.1 \pm 1.6$ & $8.1 \pm 0.9$ & 0.004 \\
\hline$\Delta \mathrm{NC}_{90}, \mathrm{~cm}$ & $0.5 \pm 0.4$ & $0.7 \pm 0.3$ & $0.4 \pm 0.3$ & 0.013 \\
\hline$\Delta \mathrm{NFV}_{90}, \mathrm{ml}$ & $15.7 \pm 4.4$ & $16.8 \pm 5.2$ & $14.3 \pm 3.1$ & 0.082 \\
\hline$\Delta \mathrm{UAXSA}_{90}, \mathrm{~cm}^{2}$ & $-0.3 \pm 0.7$ & $-0.4 \pm 0.7$ & $-0.2 \pm 0.7$ & 0.136 \\
\hline
\end{tabular}


outcome variables, women experienced a smaller change in $\mathrm{NC}$ after 90 minutes supine compared to men $(\mathrm{p}=0.013)$. There was also a tendency for an increased change in NFV after 90 minutes supine in men compared to women $(p=0.087)$. However, the differences between women and men in the change in UAXSA after 90 minutes supine was not statistically significant.

Individual correlations between baseline variables and outcome variables are summarized in Table 2 . Three baseline variables were significantly correlated with the change in NC after 90 minutes supine: $\operatorname{sex}(r=0.56, p=0.001)$, and baseline LFV at standing $(r=0.48, p=0.014)$ and supine $(r=0.51, p=0.008)$.

These correlations demonstrate that male sex and increased baseline LFV corresponded to an increase in NC over the 90-minute supine period. Change in NFV after lying supine for 90 minutes was directly and significantly correlated with baseline $\mathrm{NFV}$ at standing $(\mathrm{r}=0.41, \mathrm{p}=0.026)$ and baseline $\mathrm{UAXSA}(\mathrm{r}=0.38, \mathrm{p}=0.042)$. It was hardly significant with NFV at supine $(r=0.36, p=0.052)$. These same variables were also significantly correlated to the narrowing in the UAXSA after lying supine $\left(\mathrm{NFV}_{\mathrm{st}}\right.$ : $\left.\mathrm{r}=-0.44, \mathrm{p}=0.018, \mathrm{NFV}_{\mathrm{sp} 1}: \mathrm{r}=-0.46, \mathrm{p}=0.011, \mathrm{UAXSA}_{\mathrm{sp} 1}: \mathrm{r}=-0.48, \mathrm{p}=0.0078\right)$. Lastly, DLFV ${ }_{90}$ was significantly correlated with baseline LFV at standing $(r=0.39$, $\mathrm{p}=0.032)$ and supine $(\mathrm{r}=0.44, \mathrm{p}=0.014)$. In terms of the correlation across outcome variables, the change in $\mathrm{NC}$ was significantly correlated with the change in NFV after

Table 2. Correlation coefficients for both the individual baseline variables and the multiple linear models developed from stepwise regression

\begin{tabular}{|c|c|c|c|}
\hline \multirow[b]{2}{*}{ Variables } & \multicolumn{3}{|c|}{ Correlation } \\
\hline & $\Delta \mathrm{NC}_{90}$ & $\Delta \mathrm{NFV}_{90}$ & ${ }_{\triangle} \mathbf{U A X S A}_{90}$ \\
\hline Sex & $-0.561^{*}$ & -0.280 & 0.191 \\
\hline BMI & 0.106 & 0.144 & 0.068 \\
\hline Weight & 0.230 & 0.124 & -0.112 \\
\hline Height & 0.241 & 0.288 & -0.206 \\
\hline Age & 0.263 & -0.229 & 0.066 \\
\hline $\mathrm{NC}_{\mathrm{sp} 1}$ & 0.314 & 0.089 & -0183 \\
\hline $\mathrm{UAXSA}_{\mathrm{sp} 1}$ & -0.022 & $0.380^{*}$ & $-0.484^{*}$ \\
\hline $\mathrm{NFV}_{\text {st5 }}$ & -0.046 & $0.406^{*}$ & $-0.437^{*}$ \\
\hline $\mathbf{L F V}_{\text {st5 }}$ & $0.483^{*}$ & 0.281 & -0.002 \\
\hline$\Delta \mathbf{N F V}_{\mathbf{P}}$ & -0.346 & -0.167 & 0.083 \\
\hline$\Delta \mathbf{L F V}_{\mathbf{P}}$ & -0.091 & 0.002 & -0.176 \\
\hline $\mathrm{NFV}_{\text {sp1 }}$ & -0.106 & $0.357^{\cup}$ & $-0.463^{*}$ \\
\hline $\mathbf{L F V}_{\text {sp1 }}$ & $0.510^{*}$ & 0.292 & 0.014 \\
\hline$\Delta \mathbf{L F V}_{90}$ & 0.348 & 0.306 & 0.069 \\
\hline $\mathbf{L}_{\mathbf{U A}}$ & 0.160 & 0.121 & -0.281 \\
\hline Model & $0.687^{*}$ & $0.188^{*}$ & $0.234^{*}$ \\
\hline
\end{tabular}


Table 3. Results of the stepwise regression for each outcome variable

\begin{tabular}{lccccc}
\hline Dependent Variable & Step & Variable entered & Partial $\mathbf{R}^{2}$ & Model R $^{\mathbf{2}}$ & $\begin{array}{c}\text { P-value in the } \\
\text { final model }\end{array}$ \\
\hline$\Delta \mathrm{NC}_{90}$ & 1 & $\mathrm{Sex}$ & 0.293 & 0.293 & 0.002 \\
& 2 & $\mathrm{NFV}_{\mathrm{sp} 1}$ & 0.204 & 0.497 & $<0.001$ \\
& 3 & $\mathrm{LFV}_{\mathrm{st} 5}$ & 0.117 & 0.614 & $<0.001$ \\
& 4 & $\mathrm{NC}_{\mathrm{sp} 1}$ & 0.073 & 0.687 & $<0.001$ \\
$\Delta \mathrm{NFV}_{90}$ & 1 & $\mathrm{NFV}_{\mathrm{sp} 1}$ & 0.188 & 0.188 & 0.020 \\
$\Delta \mathrm{UAXSA}_{90}$ & 1 & $\mathrm{UAXSA}_{\mathrm{sp} 1}$ & 0.234 & 0.234 & 0.008 \\
\hline
\end{tabular}

90 minutes supine $(r=0.364, p=0.047)$. It was not correlated with the change UA-XSA after 90 minutes supine $(\mathrm{p}=0.82)$. In addition, the correlation between change in NFV and the change in UA-XSA after 90 minutes supine was not statistically significant ( $\mathrm{r}=-0.323, \mathrm{p}=0.087)$.

The linear regression models developed using stepwise feature selections for each outcome variable are summarized in Table 3 . Models for $\Delta \mathrm{NFV}_{90}$ and $\triangle \mathrm{UAXSA}_{90}$ only included one variable $\left(\mathrm{NFV}_{\mathrm{sp} 1}\right.$ and $\mathrm{UAXSA}_{\mathrm{sp} 1}$, respectively). The linear regression model of $\Delta \mathrm{NC}_{90}$ showed that sex, $\mathrm{NFV}_{\mathrm{sp} 1}, \mathrm{LFV}_{\mathrm{st} 5}$, and $\mathrm{NC}_{\mathrm{sp1}}$ contributed to the increased NC after lying supine for 90 minutes. Models developed with weight and height replacing BMI in the baseline variable set did not change the models and were not reported. The correlation between the linear regression models and the outcome variables are listed in Table 2. Since the models developed for $\triangle \mathrm{NFV}_{90}$ and $\triangle \mathrm{UAXSA}_{90}$ included only one variable, the correlations were unchanged compared to those from single-variable models. On the other hand, the correlation of the proposed model with $\Delta \mathrm{NC}_{90}$ was higher than the correlations of individual variables with $\Delta \mathrm{NC}_{90}$.

Only the first principle component from the PCA was retained, since the point of inflexion was positioned at the second component (Figure 2a). The first principle component explained $37.1 \%$ of the variance in the data set. The point of inflexion in the modified Scree plot of the first principal component (Figure $2 b$ ) was positioned around BMI. Thus, sex, baseline NC, and baseline NFV/LFV at both standing and supine positions were retained for further analysis. Given the co-linearity of the standing and supine measures of LFV and NFV, only standing LFV and NFV were included in the variable subset. Clustering based on these variables yielded two distinct groups. The dominant factor that separated the clusters was sex, so cluster 1 contained only men, while cluster 2 contained only women. To explore the data further, sex was removed from the variable subset and K-means clustering was repeated.

These results are illustrated in Figure 3. As shown in Figure 3, clusters 1 and 2 are still separated based mostly on sex, with cluster 2 consisting of only women, and cluster 1 consisting of majority men and two women. The clusters show a good separation across all of the input variables $\mathrm{NC}_{\mathrm{sp} 1}, \mathrm{NFV}_{\mathrm{st} 5}$, and $\mathrm{LFV}_{\mathrm{st} 5}$, which were significantly different between clusters (see Table 4). Differences in the value of the outcome variables of $\Delta \mathrm{NC}_{90}, \Delta \mathrm{NFV}_{90}$, and $\Delta \mathrm{UAXSA}_{90}$ between clusters 1 and 2 are shown in 

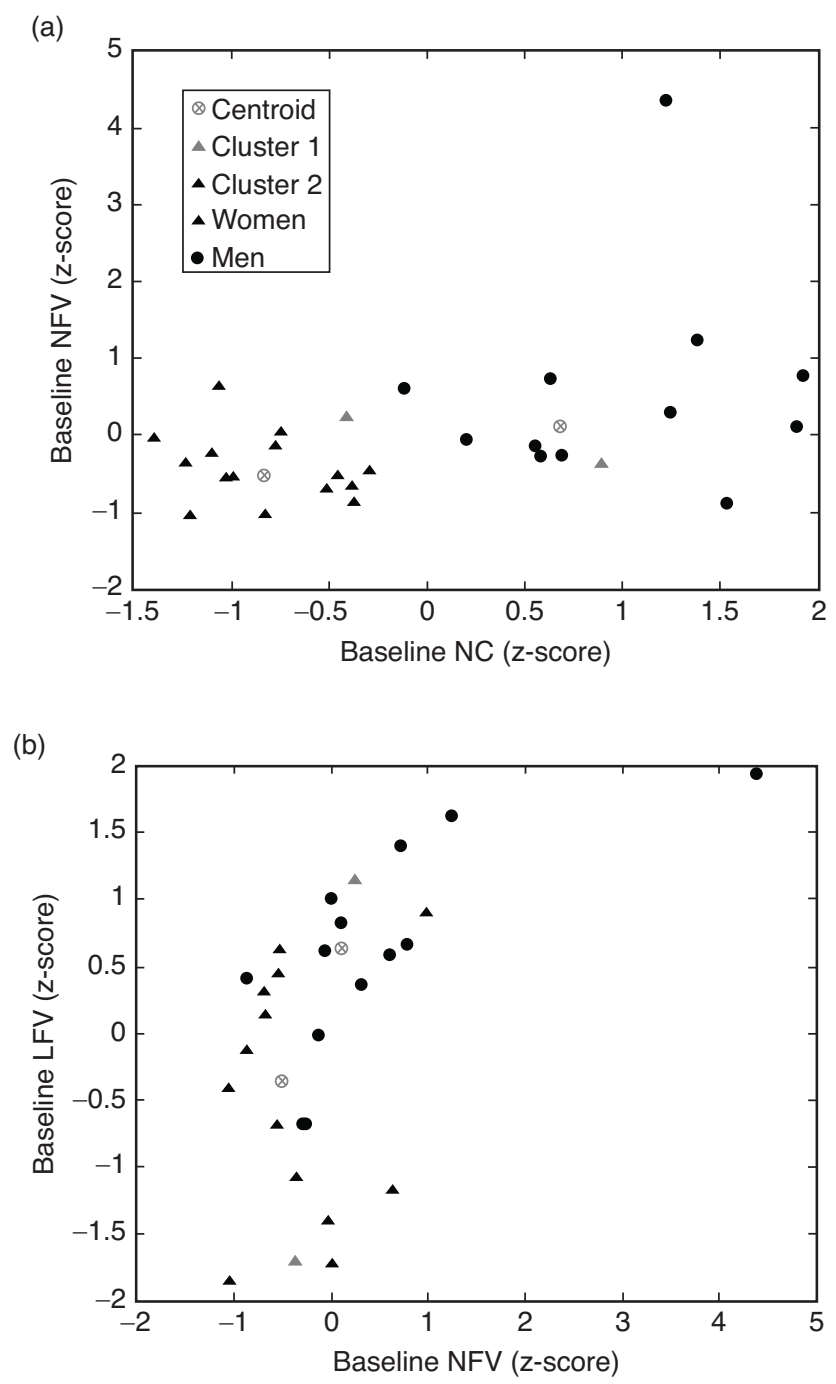

Figure 3. Plots of clusters and centroid locations on the two-dimensional plot of (a) baseline neck fluid volume (NFV) and baseline neck circumference (NC), and (b) baseline leg fluid volume (LFV) and baseline neck fluid volume (NFV). Clustering based on the variable subset that included baselines $\mathrm{NC}\left(\mathrm{NC}_{\mathrm{sp} 1}\right)$, baseline standing $\mathrm{LFV}\left(\mathrm{LFV}_{\mathrm{st} 5}\right)$, and $\mathrm{NFV}$ $\left(\mathrm{NFV}_{\mathrm{st} 5}\right)$ yielded two distinct clusters, with cluster 2 consisting of only women, and cluster 1 consisting of majority men. The input variables $\mathrm{NC}_{\mathrm{sp} 1}, \mathrm{NFV}_{\mathrm{st} 5}$, and $\mathrm{LFV}_{\mathrm{st} 5}$, were significantly different between clusters, but not the outcome variables including the change in $\mathrm{NC}, \mathrm{NFV}$ and upper-airway cross-sectional area after 90 minutes supine $\left(\Delta \mathrm{NC}_{90}\right.$, $\triangle \mathrm{NFV}_{90}$ and $\triangle \mathrm{UAXSA}_{90}$, respectively). 
Table 4. Mean and standard deviation of the variables within each cluster and associated p-values for statistical differences between the clusters.

\begin{tabular}{lccc}
\hline & Cluster 1 & Cluster 2 & P-value \\
\hline $\mathbf{N F V}_{\text {st5 }}$ & $249.7 \pm 73.4$ & $198.1 \pm 26.4$ & 0.017 \\
$\mathbf{L F V}_{\text {st5 }}$ & $2341 \pm 276$ & $2050 \pm 225$ & 0.005 \\
$\mathbf{N C}_{\text {sp1 }}$ & $39.7 \pm 2.6$ & $33.5 \pm 1.3$ & $<0.001$ \\
$\mathbf{N C C}_{\mathbf{9 0}}$ & $0.64 \pm 0.40$ & $0.40 \pm 0.28$ & 0.136 \\
$\mathbf{\Delta N F V}_{\mathbf{9 0}}$ & $17.0 \pm 5.2$ & $14.4 \pm 3.1$ & 0.308 \\
$\mathbf{\Delta} \mathbf{U X X S A}_{\mathbf{9 0}}$ & $-0.43 \pm 0.67$ & $-0.18 \pm 0.76$ & 0.148
\end{tabular}

Table 4. There was a non-significant tendency for the participants of cluster 2 to have a higher increase in $\Delta \mathrm{NC}_{90}, \Delta \mathrm{NFV}_{90}$ and a greater reduction in $\triangle \mathrm{UAXSA}_{90}$ compared to cluster 1 .

\section{DISCUSSION}

The most important finding of our study was that demographic information and baseline measures of fluid in the body could be used to predict the amount of fluid leaving the legs and the increase in neck circumference after lying down for 90 minutes. For the first time, we showed that baseline standing and supine LFV have a positive and significant correlation with changes in LFV and NC after lying supine for 90 minutes. Previous studies have shown similar findings that subjective clinical measures of leg edema score before sleep is correlated with the overnight change in LFV and increased severity of sleep apnea $[11,21]$. It has also been shown that the change in LFV during sleep is the strongest predictor of the change in $\mathrm{NC}$, as well as the consequent increase in the severity of sleep apnea [2,21]. These studies show strong evidence of the importance of the overnight change in LFV as a predictor of fluid accumulation in the neck and sleep apnea severity. However, they are based on measurements of LFV acquired overnight or during a prolonged period of recumbency. Our results are unique to suggest that a simpler objective measure of LFV taken at baseline could be used as a strong predictor of the amount of fluid that will leave the legs, causing an increase in $\mathrm{NC}$ during a prolonged period of recumbency.

Another demographic that predicted the change in NC was sex. Although change in LFV was similar between the sexes, men experienced a greater increase in the NC during a supine period. The effect of sex on rostral fluid shift has been previously shown in overnight studies in patients with heart failure. These studies showed that the overnight change in LFV in men was significantly correlated to the increase in NC [11]. For women with the same amount of fluid leaving the legs, an overnight change in LFV did not correlate with the overnight change in NC [11]. As such, men with heart failure experienced an overnight increase in neck circumference seven times greater than women [11]. Recently, Yadollahi et al. studied the dynamics of fluid shift 
in men and women lying awake in the supine position for 90 minutes to investigate the differences in fluid shift between the two sexes [34]. They found that men and women experienced a similar volume of fluid shift out of their legs, with men accumulating more fluid in their thorax and neck [34]. In addition, there was also a somewhat significant tendency for women to accumulate more fluid in their abdomen, compared to men [34]. It has been suggested that since women have larger gonadal veins and the large venous plexus around the uterus $[39,40]$, the venous pooling in the pelvic region of women is greater than that of men [41]. Therefore, in women, the fluid shifting out of the legs may be accumulating more in the pelvis and reducing fluid redistribution into the neck $[11,34]$.

Our results comply with previous studies showing that sex has a significant effect on the change in NC after 90 minutes. Clustering based on the subset of variables selected from the PCA separated the data mainly based on sex. Even when sex was excluded from the clustering analysis, the data was grouped based on baseline $\mathrm{NC}$ and baseline LFV and NFV (significant contributors to the first PCA), which were significantly different between the sexes (Table 1). In Figure 3, cluster 1 represents female subjects with lower baseline NC, NFV and LFV, whereas cluster 2 represents male subjects with higher baseline NC, NFV, and LFV. However, the stepwise regression model shows that in addition to sex, $\mathrm{LFV}_{\mathrm{st} 5}, \mathrm{NFV}_{\mathrm{sp} 1}$, and $\mathrm{NC}_{\mathrm{sp} 1}$ also entered the model. Together, these factors accounted for $40 \%$ of variation in $\Delta \mathrm{NC}_{90}$, as indicated by the partial $\mathrm{R}^{2}$ due to these factors (Table 3). The inclusion of these variables, specifically baseline LFV, demonstrates that baseline LFV has an independent effect on $\Delta \mathrm{NC}_{90}$, beyond the effects of sex. This complies with previous studies showing that interventions reducing baseline LFV, such as compression stockings [42-44], exercise [45], and diuretics [46], have led to less overnight increases in NC.

The change in NC during the supine period was also correlated with the change in $\mathrm{NFV}$, demonstrating that the change in $\mathrm{NC}$ is indeed a reflection of the change in fluid accumulating in the neck. This finding has been established by the previous work of Yadollahi et al. [34]. The correlation between $\Delta \mathrm{NFV}_{90}$ and $\triangle \mathrm{UAXSA}_{90}$ was not significant, but there was a trend to suggest that more fluid entering the neck during the supine period corresponded to further narrowing of the UA-XSA $(\mathrm{p}=0.087)$. One likely explanation for the lack of correlation between these variables is the short duration (90 minutes), during which participants are laying supine. This did not allow enough time for the airway to narrow as a result of neck fluid accumulation.

Variables that were found to be predictive of both $\triangle \mathrm{NFV}_{90}$ and $\triangle \mathrm{UAXSA}_{90}$ were baseline UAXSA and baseline NFV (standing and supine). These variables formed positive relationships with $\Delta \mathrm{NFV}_{90}$ and negative relationships with $\triangle \mathrm{UAXSA}_{90}$. Therefore, increased baseline NFV and UAXSA were related to an increased neck fluid volume and further narrowing of the upper-airway over the 90-minute supine period, both of which signal the accumulation of fluid in the neck. A likely explanation is that a higher baseline NFV and a narrower baseline UAXSA could be symptoms of having a higher overall fluid retention at baseline. Thus, when lying supine, those with a greater baseline fluid retention experience an increased fluid shift, resulting in greater increases in NFV and a narrowing of UAXSA over the 90-minute period. 
The results of this study identify characteristics that could possibly foresee those at risk of OSA through the fluid shift mechanism. Past studies have shown that an increased overnight fluid shift from the legs and an increased overnight change in NC are both strongly related to an increased OSA severity [2]. This study identifies phenotypic characteristics, specifically of the male sex, and increased baseline LFV, all of which are related to both an increased fluid shift from the legs and an increased change in $\mathrm{NC}$ when lying supine for a prolonged period, therefore possibly predicting the likelihood of OSA through the fluid shift mechanism. Future work is aimed at exploring the capability of these characteristics and predicting those at risk of OSA through the fluid shift mechanism.

This study is subject to limitation, mainly imposed by the sensitivity of bioelectrical impedance measurements to movement. Bioelectrical impedance measurements are sensitive to movement and body posture, and because our participants could not remain still for more than 90 minutes, the duration of study was limited to just that. As a result, the fluid shift might be scaled down compared to overnight fluid shift. Our study was performed during wakefulness to limit involuntary body movements during sleep. Since the pattern of fluid redistribution out of the legs and into the neck could vary depending on whether or not the subject was awake or sleeping, future work should include studies specifically during sleep. Finally, we did not examine correlations between baseline variables and outcome variables in men and women separately due to the small number of subjects in each group. In the future we aim to confirm our findings in larger groups of men and women to gain a better understanding of fluid shift between the sexes. In addition, future work should adapt the models developed in our study using data from overnight polysomnography - the gold standard for diagnosing sleep apnea. Models developed with these data can be used to predict outcomes related to respiratory disorders affected by fluid, such as OSA.

\section{CONCLUSIONS}

In conclusion, this study demonstrates that a baseline measure LFV is predictive of the amount of fluid leaving the legs and the change in NC after lying supine for 90 minutes. Our findings also suggest that with comparable changes in LFV over the supine period, men experience greater fluid accumulation in the neck, compared to women. Applications of these findings are in identifying characteristics of individuals at risk of OSA through an overnight rostral fluid shift. There are also applications in the development of novel therapies aimed at reducing baseline LFV to minimize the associated rostral fluid shift and accumulation in the neck during sleep. Such therapies may include reducing sedentary behaviors such as prolonged sitting [14] and wearing compression stockings $[42,43]$. Future work should explore other interventions such as physical activity $[45,47,48]$ or electrical stimulation of the calf muscle pump [49] to reduce baseline leg edema and neck fluid accumulation in a population at risk for fluid shift related sleep apnea.

\section{CONFLICT OF INTEREST}

The authors indicated no potential conflicts of interest. 


$\begin{array}{ll}\text { ABBREVIATIONS } \\ \text { BMI } & \text { Body mass index } \\ \mathrm{NC}_{\text {sp1 }} & \text { Neck circumference at } 1 \text { minute supine } \\ \mathrm{UAXSA}_{\text {sp1 } 1} & \text { Upper airway cross-sectional area at } 1 \text { minute supine } \\ \mathrm{NFV}_{\mathrm{st5}} & \text { Neck fluid volume at } 5 \text { minutes standing } \\ \mathrm{LFV}_{\mathrm{st5}} & \text { Leg fluid volume at } 5 \text { minutes standing } \\ \Delta \mathrm{NFV} & \text { Change in neck fluid volume due to posture change } \\ \Delta \mathrm{LFV}_{\mathrm{P}} & \text { Change in leg fluid volume due to posture change } \\ \mathrm{NFV}_{\mathrm{sp} 1} & \text { Neck fluid volume at } 1 \text { minute supine } \\ \mathrm{LFV}_{\mathrm{sp} 1} & \text { Leg fluid volume at } 1 \text { minute supine } \\ \Delta \mathrm{LFV}_{90} & \text { Change in leg fluid volume after } 90 \text { minutes supine } \\ \Delta \mathrm{NC} & \text { Change in neck circumference after } 90 \text { minutes supine } \\ \Delta \mathrm{NFV} & \text { Change in neck fluid volume after } 90 \text { minutes supine } \\ \Delta \mathrm{UAXSA}_{90} & \text { Change in upper airway cross-sectional area after } 90 \text { minutes supine } \\ \mathrm{L}_{\mathrm{UA}} & \text { Length of the upper airway }\end{array}$

\section{REFERENCES}

[1] K. L. Chiu, C. M. Ryan, S. Shiota, P. Ruttanaumpawan, M. Arzt, J. S. Haight, C. T. Chan, J. S. Floras, and T. D. Bradley. Fluid shift by lower body positive pressure increases pharyngeal resistance in healthy subjects. Am. J. Respir. Crit. Care Med., 2006, 174(12):1378-1383.

[2] S. Redolfi, D. Yumino, P. Ruttanaumpawan, B. Yau, M. C. Su, J. Lam, and T. D. Bradley. Relationship between overnight rostral fluid shift and Obstructive Sleep Apnea in nonobese men. Am. J. Respir. Crit. Care Med., 2009, 179(3): 241-246.

[3] S. Shiota, C. M. Ryan, K. L. Chiu, P. Ruttanaumpawan, J. Haight, M. Arzt, J. S. Floras, C. Chan, and T. D. Bradley. Alterations in upper airway cross-sectional area in response to lower body positive pressure in healthy subjects. Thorax, 2007, 62(10):868-872.

[4] M. C. Su, K. L. Chiu, P. Ruttanaumpawan, S. Shiota, D. Yumino, S. Redolfi, J. S. Haight, and T. D. Bradley. Lower body positive pressure increases upper airway collapsibility in healthy subjects. Respir Physiol Neurobiol, 2008, 161(3):306-312.

[5] O. Friedman, T. D. Bradley, and A. G. Logan. Influence of lower body positive pressure on upper airway cross-sectional area in drug-resistant hypertension. Hypertension, 2013, 61(1):240-245.

[6] J. W. Shepard, D. A. Pevernagie, A. W. Stanson, B. K. Daniels, and P. F. Sheedy. Effects of changes in central venous pressure on upper airway size in patients with obstructive sleep apnea. Am. J. Respir. Crit. Care Med., 1996, 153(1):250-254.

[7] T. Kasai, S. S. Motwani, D. Yumino, J. M. Gabriel, L. T. Montemurro, V. Amirthalingam, J. S. Floras, and T. D. Bradley. Contrasting effects of lower body positive pressure on upper airways resistance and partial pressure of carbon dioxide in men with heart failure and obstructive or central sleep apnea. $J$. Am. Coll. Cardiol., 2013, 61(11):1157-1166.

[8] M. C. Su, K. L. Chiu, P. Ruttanaumpawan, S. Shiota, D. Yumino, S. Redolfi, J. S. Haight, B. Yau, J. Lam, and T. D. Bradley. Difference in upper airway collapsibility during wakefulness between men and women in response to lower-body positive pressure. Clin. Sci., 2009, 116(9):713-720.

[9] L. H. White and T. D. Bradley. Role of nocturnal rostral fluid shift in the pathogenesis of obstructive and central sleep apnoea. J. Physiol.(Lond.), 2013, 591(5):1179-1193.

[10] O. Friedman, T. D. Bradley, C. T. Chan, R. Parkes, and A. G. Logan. Relationship between overnight rostral fluid shift and obstructive sleep apnea in drug-resistant hypertension. Hypertension, 2010, 56(6):1077-1082. 
[11] T. Kasai, S. S. Motwani, D. Yumino, S. Mak, G. E. Newton, and T. D. Bradley. Differing relationship of nocturnal fluid shifts to sleep apnea in men and women with heart failure. Circ Heart Fail, 2012, 5(4):467-474.

[12] E. H. Starling. On the Absorption of Fluids from the Connective Tissue Spaces. J. Physiol. (Lond.), 1986, 19(4):312-326.

[13] A. Krogh, E. M. Landis, and A. H. Turner. The movement of fluid throguh the human capillary wall in relation to venous pressure and to the collogid osmotic pressure of the blood. J. Clin. Invest., 1932, 11(1):63-95.

[14] M. Pottier, A. Dubreuil, and H. Monod. The effects of sitting posture on the volume of the foot. Ergonomics, 1969, 12(5):753-758.

[15] R. L. Waterfield. The effect of posture on the volume of the leg. J Physiol, 1931, 72(1):121-131.

[16] J. B. Youmans, H. S. Wells, D. Donley, D. G. Miller, and H. Frank. The effect of posture (standing) on the serum protein concentration and colloid osmotic pressure of blood from the foot in relation to the formation of edema. J. Clin. Invest., 1934, 13(3):447-459.

[17] P. Avasthey and E. H. Wood. Intrathoracic and venous pressure relationships during responses to changes in body position. J Appl Physiol, 1974, 37(2):166-175.

[18] G. Baccelli, P. Pacenti, S. Terrani, M. Checchini, G. Riglietti, F. Prestipino, E. Omboni, F. Sardella, M. Catalano, and Z. Malacarne. Scintigraphic recording of blood volume shifts. J. Nucl. Med., 1995, 36(11): 2022-2031.

[19] W. Hildebrandt, H. C. Gunga, J. Herrmann, L. Rocker, K. Kirsch, and J. Stegemann, Enhanced slow caudad fluid shifts in orthostatic intolerance after 24-h bed-rest, Eur J Appl Physiol Occup Physiol, 1994, 69(1):61-70.

[20] N. Terada and T. Takeuchi, Postural changes in venous pressure gradients in anesthetized monkeys. Am. J. Physiol, 1993, 264(1):H21-25.

[21] D. Yumino, S. Redolfi, P. Ruttanaumpawan, M. C. Su, S. Smith, G. E. Newton, S. Mak, and T. D. Bradley. Nocturnal rostral fluid shift: a unifying concept for the pathogenesis of obstructive and central sleep apnea in men with heart failure. Circulation, 2010, 121(14):1598-1605.

[22] B. E. Schroth. Evaluation and management of peripheral edema. JAAPA, 2005, 18(11):29-34.

[23] A. Yadollahi, F. Rudzicz, S. Mahallati, M. Coimbra, and T. D. Bradley. Acoustic Estimation of Neck Fluid Volume. Ann Biomed Eng, 2014, 42(10):2132-2142.

[24] J. J. Fredberg, M. E. Wohl, G. M. Glass, and H. L. Dorkin. Airway area by acoustic reflections measured at the mouth. J Appl Physiol Respir Environ Exerc Physiol, 1980, 48(5):749-758.

[25] S. Demura, S. Sato, and T. Kitabayashi. Percentage of total body fat as estimated by three automatic bioelectrical impedance analyzers. J Physiol Anthropol Appl Human Sci, 2004, 23(3):93-99.

[26] F. Zhu, D. Schneditz, E. Wang, and N. W. Levin. Dynamics of segmental extracellular volumes during changes in body position by bioimpedance analysis. J. Appl. Physiol., 1998, 85(2):497-504.

[27] M. Y. Jaffrin and H. Morel. Body fluid volumes measurements by impedance: A review of bioimpedance spectroscopy (BIS) and bioimpedance analysis (BIA) methods. Med Eng Phys, 2008, 30(10):1257-1269.

[28] U. G. Kyle, I. Bosaeus, A. D. De Lorenzo, P. Deurenberg, M. Elia, J. M. Gomez, B. L. Heitmann, L. Kent-Smith, J. C. Melchior, M. Pirlich, H. Scharfetter, A. M. Schols, and C. Pichard. Bioelectrical impedance analysis-part I: review of principles and methods. Clin Nutr, 2004, 23(5):1226-1243.

[29] A. De Lorenzo, A. Andreoli, J. Matthie, and P. Withers. Predicting body cell mass with bioimpedance by using theoretical methods: a technological review. J. Appl. Physiol., 1997 82(5):1542-1558.

[30] D. Bracco, D. Thiebaud, R. L. Chiolero, M. Landry, P. Burckhardt, and Y. Schutz. Segmental body composition assessed by bioelectrical impedance analysis and DEXA in humans. J. Appl. Physiol., 1996, 81(6):2580-2587.

[31] C. H. Ling, A. J. de Craen, P. E. Slagboom, D. A. Gunn, M. P. Stokkel, R. G. Westendorp, and A. B. Maier. Accuracy of direct segmental multi-frequency bioimpedance analysis in the assessment of total 
body and segmental body composition in middle-aged adult population. Clin Nutr, 2011, 30(5):610-615.

[32] A. Tagliabue, A. Andreoli, M. Comelli, S. Bertoli, G. Testolin, G. Oriani, and A. De Lorenzo. Prediction of lean body mass from multifrequency segmental impedance: influence of adiposity. Acta Diabetol, 2001, 38(2):93-97.

[33] F. Zhu, M. K. Kuhlmann, P. Kotanko, E. Seibert, E. F. Leonard, and N. W. Levin. A method for the estimation of hydration state during hemodialysis using a calf bioimpedance technique. Physiol Meas, 2008, 29(6):S503-516.

[34] A. Yadollahi, B. Singh, and T. D. Bradley. Investigating the Dynamics of Supine Fluid Redistribution Within Multiple Body Segments Between Men and Women. Ann Biomed Eng, 2015, 43(9):2131-2142

[35] M. Fenech and M. Y. Jaffrin. Extracellular and intracellular volume variations during postural change measured by segmental and wrist-ankle bioimpedance spectroscopy. IEEE Trans Biomed Eng, 2004, 51(1):166-175.

[36] XITRON, HYDRA ECF/ICF (Model 4200), Bio-impedance spectrum analyzer, For measuring intracellular and extracellular fluid volumes. Operating manual. XITRON Technologies Inc., 2007.

[37] I. Jolliffe, Principal component analysis. Wiley Online Library, 2002.

[38] J. A. Hartigan and M. A. Wong. A k-means clustering algorithm: Algorithm AS 136. Applied statistics, 1979, 28(1):100-108.

[39] N. E. Ahlberg, O. Bartley, and N. Chidekel. Right and left gonadal veins. An anatomical and statistical study. Acta Radiol Diagn (Stockh), 1966, 4(6):593-601.

[40] H. Gray, The veins of the abdomen and pelvis, in Anatomy of the Human Body, 29th ed., C. Goss, Ed. Philadelphia, PA: Lea \& Febiger, 1973, 709-715.

[41] D. D. White and L. D. Montgomery. Pelvic blood pooling of men and women during lower body negative pressure. Aviat Space Environ Med, 1996, 67(6):555-559.

[42] S. Redolfi, I. Arnulf, M. Pottier, J. Lajou, I. Koskas, T. D. Bradley, and T. Similowski. Attenuation of obstructive sleep apnea by compression stockings in subjects with venous insufficiency. Am.J. Respir. Crit. Care Med., 184(9):1062-1066.

[43] S. Redolfi, I. Arnulf, M. Pottier, T. D. Bradley, and T. Similowski. Effects of venous compression of the legs on overnight rostral fluid shift and obstructive sleep apnea. Respir Physiol Neurobiol, 2011, 175(3):390-393.

[44] L. H. White, O. D. Lyons, A. Yadollahi, C. M. Ryan, and T. D. Bradley, Effect of below-the-knee compression stockings on severity of obstructive sleep apnea, Sleep Med., 2015, 16(2):258-264.

[45] S. Redolfi, M. Bettinzoli, N. Venturoli, M. Ravanelli, L. Pedroni, L. Taranto-Montemurro, I. Arnulf, T. Similowski, and C. Tantucci. Attenuation of obstructive sleep apnea and overnight rostral fluid shift by physical activity. Am. J. Respir. Crit. Care Med., 2015, 191(7):856-858.

[46] T. Kasai, T. D. Bradley, O. Friedman, and A. G. Logan. Effect of intensified diuretic therapy on overnight rostral fluid shift and obstructive sleep apnoea in patients with uncontrolled hypertension. J. Hypertens., 2014, 32(3):673-680.

[47] I. H. Iftikhar, C. E. Kline, and S. D. Youngstedt. Effects of exercise training on sleep apnea: a metaanalysis. Lung, 2014, 192(1):175-184.

[48] C. E. Kline, E. P. Crowley, G. B. Ewing, J. B. Burch, S. N. Blair, J. L. Durstine, J. M. Davis, and S. D. Youngstedt. The effect of exercise training on obstructive sleep apnea and sleep quality: a randomized controlled trial. Sleep, 2011, 34(12):1631-1640.

[49] A. A. Goddard, C. S. Pierce, and K. J. McLeod. Reversal of lower limb edema by calf muscle pump stimulation. J Cardiopulm Rehabil Prev, 2008, 28(3):174-179. 



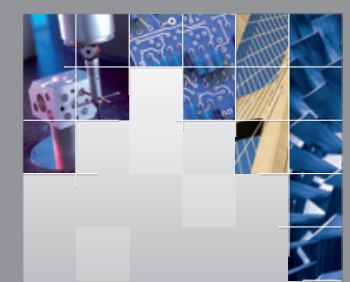

\section{Enfincering}
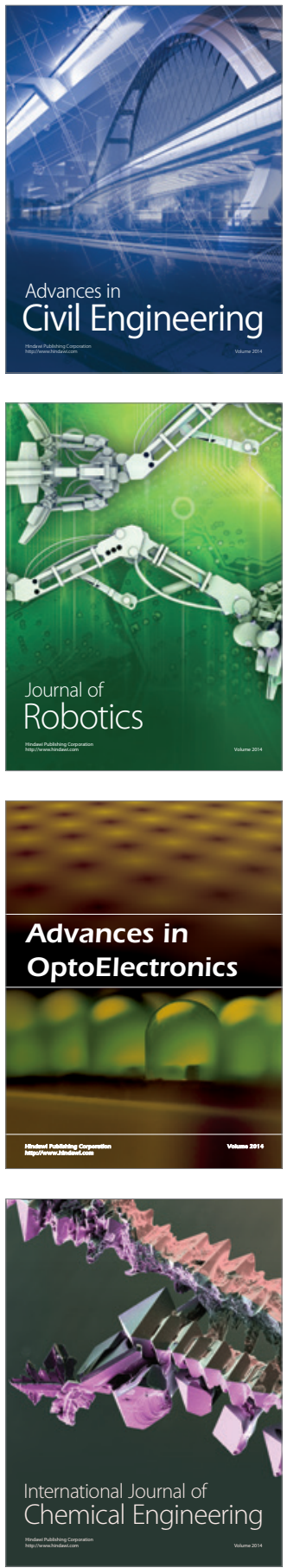

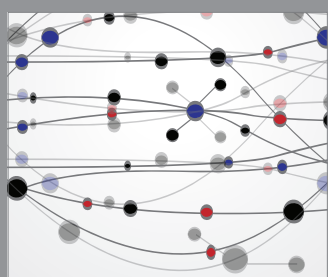

The Scientific World Journal

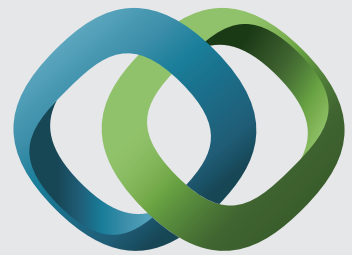

\section{Hindawi}

Submit your manuscripts at

http://www.hindawi.com
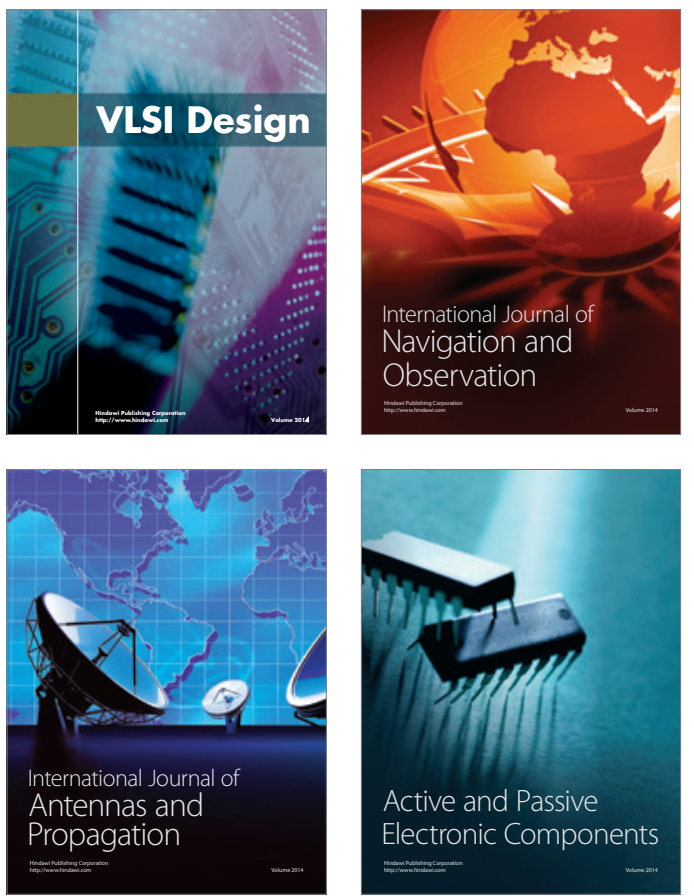
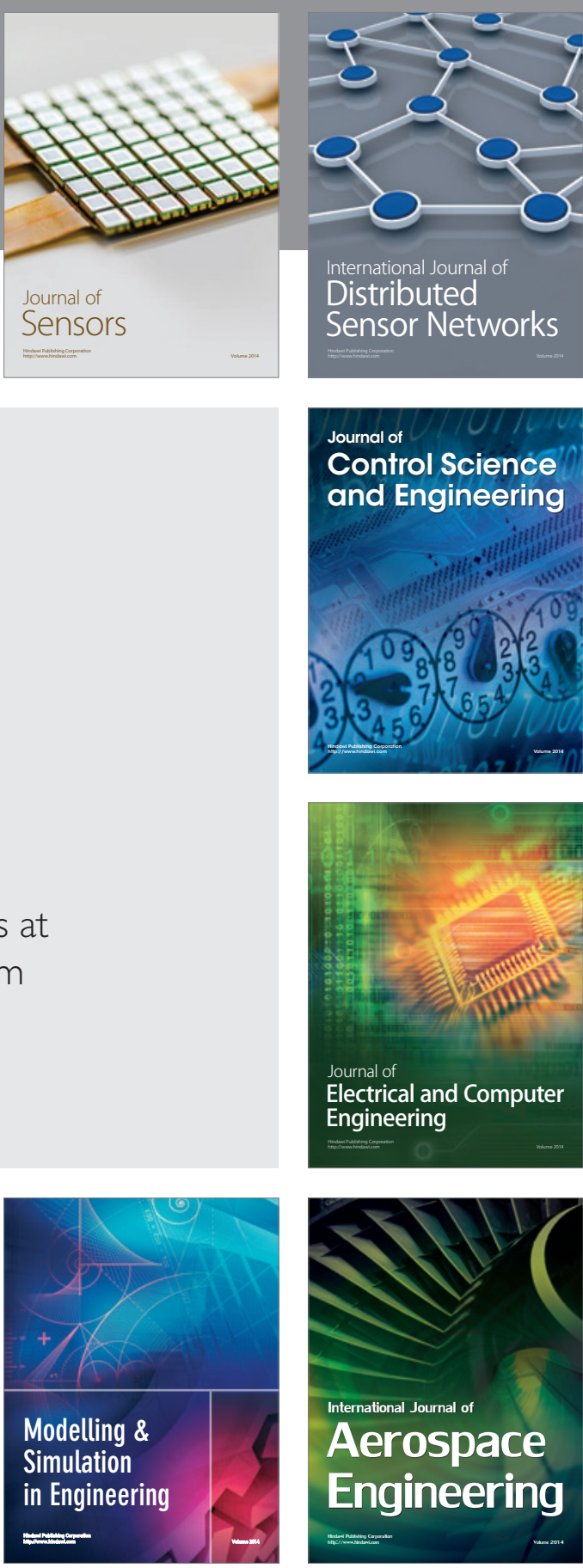

International Journal of

Distributed

Sensor Networks

Journal of

Control Science

and Engineering
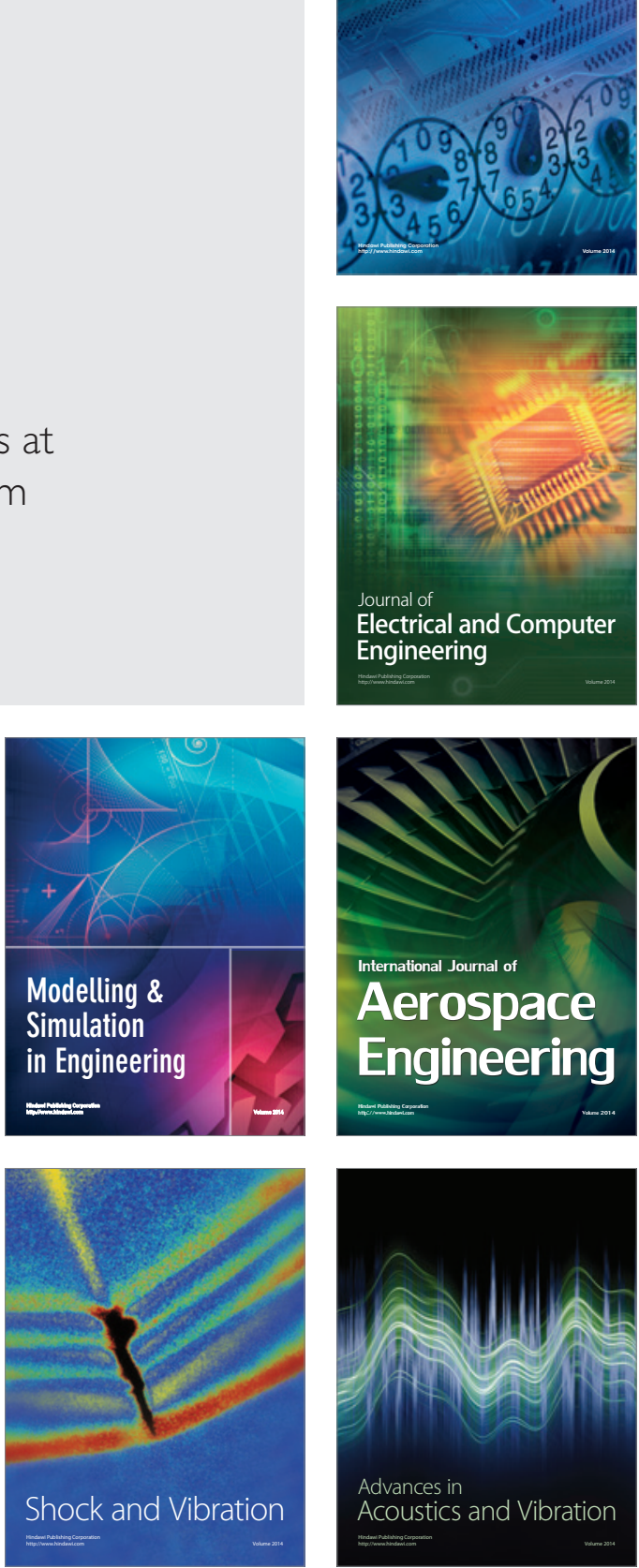